\title{
Variable Operation of Hall Thruster with Multiple Segmented Electrodes
}

\author{
N.J. Fisch, Y. Raitses, L.A. Dorf and A.A. Litvak \\ Princeton Plasma Physics Laboratory, Princeton University, Princeton NJ 08540
}

\begin{abstract}
Variable plasma jet velocity with low beam divergence over a range of mass flow rates can be achieved through segmented electrode operation of Hall plasma accelerator. With the use of just a cathode side electrode at the cathode potential, the beam divergence can be decreased substantially, at some cost in efficiency. However, the additional use of an anode side electrode retains the same reduced plume divergence, but at efficiencies comparable to the non-segmented operation. The high efficiency persists also when the anode side electrode is biased at an intermediate potential, thus producing two-stage Hall accelerator operation.
\end{abstract}




\section{Introduction}

The Hall plasma accelerator is an electrical discharge device in which a plasma jet is accelerated by a combined operation of axial electric and magnetic fields applied in a coaxial channel. Since both the ionization of the working gas atoms and the acceleration of the resulted ions take place in the same neutral electric discharge, neither process is subject to space charge limitations. Hence, Hall accelerators can provide higher jet velocities $(10 \mathrm{~km} / \mathrm{s}-20 \mathrm{~km} / \mathrm{s})$ with larger current densities $\left(\sim 0.1 \mathrm{~A} / \mathrm{cm}^{2}\right)$ than can conventional ion sources. ${ }^{1}$

The design of existing Hall accelerators, including the channel geometry, the material, and the magnetic field distribution, depends strongly on the input parameters of the accelerator, such as, the working gas, the gas flow rate, or the discharge voltage. ${ }^{2}$ Hall plasma thrusters for satellite station keeping were developed, studied and evaluated extensively for a Xenon gas propellant and jet velocities of about $15 \mathrm{~km} / \mathrm{s}$, which corresponds to a discharge voltage of $300 \mathrm{~V} .^{3,4}$ Hall thrusters of different geometry optimized for different input power levels $(0.5-10 \mathrm{~kW})$ were developed and successfully deployed on communication sattelites. ${ }^{4}$

What remains a challenge is a Hall thruster able to operate efficiently under variable conditions, such as at different input powers or at varying output thrusts. ${ }^{5}$ A number of issues arise with a variable operation of Hall current accelerators. ${ }^{6-8}$ These issues include decreased thruster efficiency, $\eta=T^{2} / 2 m P_{e}$, where $T, m$ and $P_{e}$ are the thrust, mass flow rate and the input power, respectively, for low mass flow rate and for low discharge voltage. ${ }^{6,7}$ At lower mass flow rates, the atomic density in the channel is lower, so that 
the ionization efficiency decreases ion losses increase. Moreover, an extended ionization region produces a spread of ion energies, including slow ions, which particularly contribute to enlarge the plume divergence. This is a crucial issue even for nonvariable operation. A similar effect would be incurred through the use of not easily ionized gases. $^{8}$

\section{Segmented Electrode Hall Thruster}

In conventional Hall accelerators, the key element controlling ionization and acceleration processes in the channel is the magnetic field distribution. ${ }^{1,2}$ A different control approach, which involves segmented electrodes along a dielectric channel, was suggested in Ref. 9. Fig. 1 shows a schematic view for the case of two segmented electrodes. Electrodes are placed along the channel axis on low potential side (negative side or NS electrode), as well as on the positive side (PS). If these electrodes are emissive and biased, for example by the cathode (NS) anode (PS) potentials, a significant electric field should be established across the dielectric gap between these electrodes. The accelerating electric field and, as a result, beam divergence and plume should then be sensitive to the details not only of the magnetic field distribution, but also the gap extent. Moreover, it is possible to bias these electrodes by different potentials from separate power supplies, for example, in order to separate ionization and acceleration processes in the channel.

In Ref. 9, we reported that, even if the NS segmented electrode is not emissive and floating, it can still strongly affect operation of the accelerator. The difference between segmented and non segmented accelerated cases was attributed to a difference in secondary emission coefficients of channel and electrode materials. ${ }^{10}$ Decreased plume divergence was reported in parametric studies of a Hall accelerator employing a single 
segmented electrode placed at the cathode side of the channel. ${ }^{11}$ Although the plume divergence could be substantially improved over conventional operation, particularly at low mass flow rates, the single electrode configuration entails some decrease in propellant utilization efficiency.

In order to overcome the observed reduction of the propellant utilization, a certain channel profile can be useful. Ref. 6 and 7 showed that a reduction of the channel cross section in the ionization region can increase the ionization. The increase might be due to either the increased atomic density and, as a result, ionization collisions at small mass flow rate ${ }^{6}$, or to a more uniform and focused neutral flux. ${ }^{7}$ The present study shows that the low plume divergence operation is possible without the loss in efficiency if a second anode-side segmented electrode with a certain profile is employed in addition to the cathode side electrode. That anode-side segment tends to increase the efficiency if it is biased at the anode potential, or sometimes even if it is not biased. If the anode-side segment is biased at an intermediate potential, then two-stage operation, similarly at high efficiency and low plume divergence, can be achieved.

Thus, the use of these electrodes extends considerably the parameter regimes for favorable operating characteristics of Hall plasma accelerators. Through simple switching of electrode energizing, one may achieve such a variable mode operation. The precise placement of these electrodes along the channel, as well as their precise shape and emissivity, is significant. 


\section{Experimental setup}

The experiments took place in a $28 \mathrm{~m}^{3}$ stainless steel vacuum vessel. Fig. 2 shows an experimental Hall current accelerator with two segmented electrodes. The accelerator is suspended on a $0.5 \mathrm{mN}$ resolution pendulum type thrust stand, which measures the total average jet velocity of the ion flux from the accelerator (plasma jet). An electrostatic probe, which is mounted on a rotating arm, measures the angular ion flux distribution from the accelerator. In the present set of experiments, the distance between the probe and the accelerator was $33 \mathrm{~cm}$. Since the diameter of vacuum vessel is about $2.3 \mathrm{~m}$, the probe was far enough from the walls of the vacuum vessel to minimize the influence of back flows on the probe measurements. By integrating over the angular distribution, the total ion flux and plume angle for $90 \%$ of the flux are estimated. Reproducibility of plume measurements is $\pm 4^{\circ}$ of the full angle. The propellant utilization, which is the ratio of the total ion flux to the total input flow of neutral atoms, is derived from the probe and mass flow measurements. The test facility, thruster, diagnostic setup and experimental procedure are described in greater detail in Ref. 11.

Both the anode-side and cathode-side segmented electrodes have about $1 \mathrm{~mm}$ thickness of $\mathrm{LaB}_{6}$, plated in a rhenium mesh to allow a strong structure of the emissive layer. This mesh was mounted on a molybdenum substrate ring of $3 \mathrm{~mm}$ thickness for each electrode. The length of the NS and PS electrodes was $4 \mathrm{~mm}$ and $10 \mathrm{~mm}$, respectively. The same sizes were used in Ref. 9 for Tantalum segmented electrodes.

The various locations where the electrodes might be place can be seen from Fig. 3. In the present work, the NS segmented electrode was attached to the inner wall, while PS 
electrode was inserted into the channel on its outer wall. This placement of the segmented electrodes made for easier implementation of operation with two segmented electrodes and prevented electrical breakdown along the channel wall. Note that the positive side segmented electrode has a triangle cross-section of a $5 \mathrm{~mm}$ height pointed towards the accelerator axis. The two electrode sides, which are not attached to the wall, have a $\mathrm{LaB}_{6}$ layer. Thus, this electrode reduces the channel cross section area by $33 \%$ at the peak the triangle.

The above locations of the segmented electrodes correspond to those in the previously reported investigation of the one segmented case, where electrodes are placed at either the cathode side or the anode side..$^{9,11}$ In the two-segmented electrode cases described below, the cathode-side electrode is always at position NS2. When the anode-side electrode is in position PS2, exactly $16 \mathrm{~mm}$ separates the two electrodes. When the anode-side electrode is in position PS1, exactly $10 \mathrm{~mm}$ separates the two electrodes. Accordingly, those two cases are sometimes referred to respectively as the $16 \mathrm{~mm}$ and 10 $\mathrm{mm}$ cases. Accelerator operation with segmented electrodes are compared to those measured with non segmented configuration of the accelerator referred bellow to WS.

Note, that the PS electrode has a triangular cross section with a $5 \mathrm{~mm}$ height into the channel. The $\mathrm{LaB}_{6}$ layer covers two sides of this triangle cross section ring, which are both open to the plasma. 


\section{Experimental results}

One of the features of segmented electrode operation is an increase in the discharge current when a cathode-side electrode is biased at the cathode potential. ${ }^{11}$ In the case of a single segmented electrode configuration, we attributed this increase to a reduction of the magnetic insulation as the length of the acceleration and ionization region is reduced due to low secondary emission from the metal wall of the NS electrode. Fig. 4 shows that this increase in the discharge current at gas flow rate of $1.7 \mathrm{mg} / \mathrm{s}$ persists when an anode-side electrode is inserted, whether that anode-side electrode is at floating potential or biased to the anode potential. Note that the mere presence of the segmented electrodes when the cathode-side electrode is not biased does not particularly produce an increase in the discharge current.

Figure 5 shows the propellant utilization. It is of interest that the propellant utilization is significantly increased over the conventional non-segmented case. An interesting comparison can be made also to the previously reported results for one segment only, at NS2, where no anode side segmented electrode was present. ${ }^{11}$ In the case of cathode side bias, the propellant utilization is increased by as much as $10 \%$ by the mere presence of the anode-side electrode, whether the anode-side electrode is biased or floating. The reason for this might be that the triangle-shaped anode-side electrode increases the density or possibly increases the uniformity of the density. ${ }^{6,7}$ For whatever reason, the increased propellant utilization can lead to increased performance.

The increase in discharge current accompanied by increased propellant utilization, compared to conventional operation, leads to an overall performance for two-segmented operation not worse than conventional operation. Fig. 6 gives the thrust and the thruster 
efficiency as a function of discharge voltage. Note that the one-segmented electrode case gives less thrust and poorer efficiency than both the conventional operation and the twosegment operation.

With the efficiency and thrust comparable to conventional operation, the two-segment approach is superior to the one-segment approach, so long as the favorable plume characteristics of the one-segment operation are retained. Figs. 7 show that the favorable plume characteristics are in fact largely unaffected by the presence of the second, anodeside electrode. Figs. $7 \mathrm{a}$ and $7 \mathrm{~b}$ shows the persistence of the reduction in half angle plume divergence by 10 degrees at low mass flow rates. This low mass flow rate regime is where the plume reduction tends to be greatest. ${ }^{11}$

Interestingly, the plume divergence is largely dictated by the location of the cathode-side electrode, with the anode-side electrode playing a very minor role. Yet, the anode-side electrode plays a very telling role in increased propellant utilization. The combined effect thus creates the opportunity for variable thrust at no cost in efficiency but with favorable plume divergence.

The opportunity for variable operation is much enhanced because the anode-side electrode can also be biased not necessarily at the anode potential, but at a potential intermediate between the anode and the cathode. Figure 8 show this so-called "twostage" operation, with the anode-side electrode biased $100 \mathrm{~V}$ negative with respect to the anode and $200 \mathrm{~V}$ positive with respect to the cathode. Of course, this requires a separate power supply. Note that the efficiencies are similar to conventional operation, whereas the cathode-side electrode segment can be used to control the plume divergence. 


\section{Summary}

The use of a second segmented electrode on the anode-side of the Hall thruster combines the favorable plume divergence characteristic of single-segmented operation with the high efficiency of conventional operation. The increase of the ionization efficiency at low mass flow rates might be attributed to the smaller effective channel cross sectional area in the ionization region in which the PS electrode is placed. The decrease in plume divergence is particularly significant at low mass flow rates. It appears that the added flexibility through the use of these electrodes can extend the parameter regimes for favorable operating characteristics of Hall Thrusters. Through simple switching of electrode energizing, one may achieve such a variable mode operation.

\section{Acknowledgment}

The authors thank Prof. A. Fruchtman, Dr. M. Keidar and V. Soukhanovskii for helpful discussions. The authors are indebted to R. Yager for technical support. The authors appreciate also the assistance of K. Ertmer, K-M. Fu, and E. Ortiz.

This work was supported by the U.S. DOE under Contract No. DE-ACO2-76-CHO3073. 


\section{References}

1. Zhurin, V. V., Kaufman, H. R. and Robinson, R. S., Physics of closed drift thrusters, Plasma Sources Sci. Technol, 8, 1999.

2. Bugrova A. I., Maslennikov, N., Morozov, A. I., Similarity laws for the global properties of a Hall accelrators, Sov. Phys. Tech. Phys., 36(6), June 1991.

3. King, L. B. and Gallimore, A. D., Ionic and Neutral Particle Transport Property Measurements in the Plume of an SPT-100, AIAA paper 96-2712, Lake Buena Vista, Fl, July 1996.

4. Marec, J. P., Optimal Space Trajectories, Elsevier Scientific Publishing Co. Amsterdam, 1979.

5. Martinez-Sanchez, M. and Pollard J. E., Spacecraft Electric Propulsion-An Overview, Journal of Propulsion and Power, 5, Sept.-Oct. 1998, pp. 688-699.

6. Jakupov A. B., Khartov, S. A. and Yakubov, A. M., Some results of the small power SPT models, IEPC-97-151, 25 ${ }^{\text {th }}$ International Electric Propulsion Conference, Cleveland, OH, August 1997.

7. Y. Raitses, J. Ashkenazy and M. Guelman, Propellant utilization in Hall thrusters, Journal of Propulsion and Power, 14, 1998, 247.

8. Morozov, A. I., et al, Plasma accelerator with closed electron drift and extentded acceleration zone, Sov. Phys. Tech. Phys, 17(1), 1972, pp. 38-45.

9. Fisch, N.J., Raitses, Y., Litvak, A. A., and Dorf, L. A., "Design and Operation of Hall Thrusters with Segmented Electrodes" AIAA 99-2572, Los Angeles, CA, June 1999.

10. Bugrova, A. I., Desyatkov, A. V., Morozov, A. I. And Kharchevnikov, Rearrangement of a discharge in a steady-state plasma engine due to the dusting of insulators, Plasma Physics reports, 22(4), 1996, pp. 302-305. 
11. Raitses, Y., Dorf, L. A., Litvak, A. A. and Fisch, N. J., Effect of a plume reduction in segmented electrode Hall thruster, Submitted to Journal of Applied Physics. 


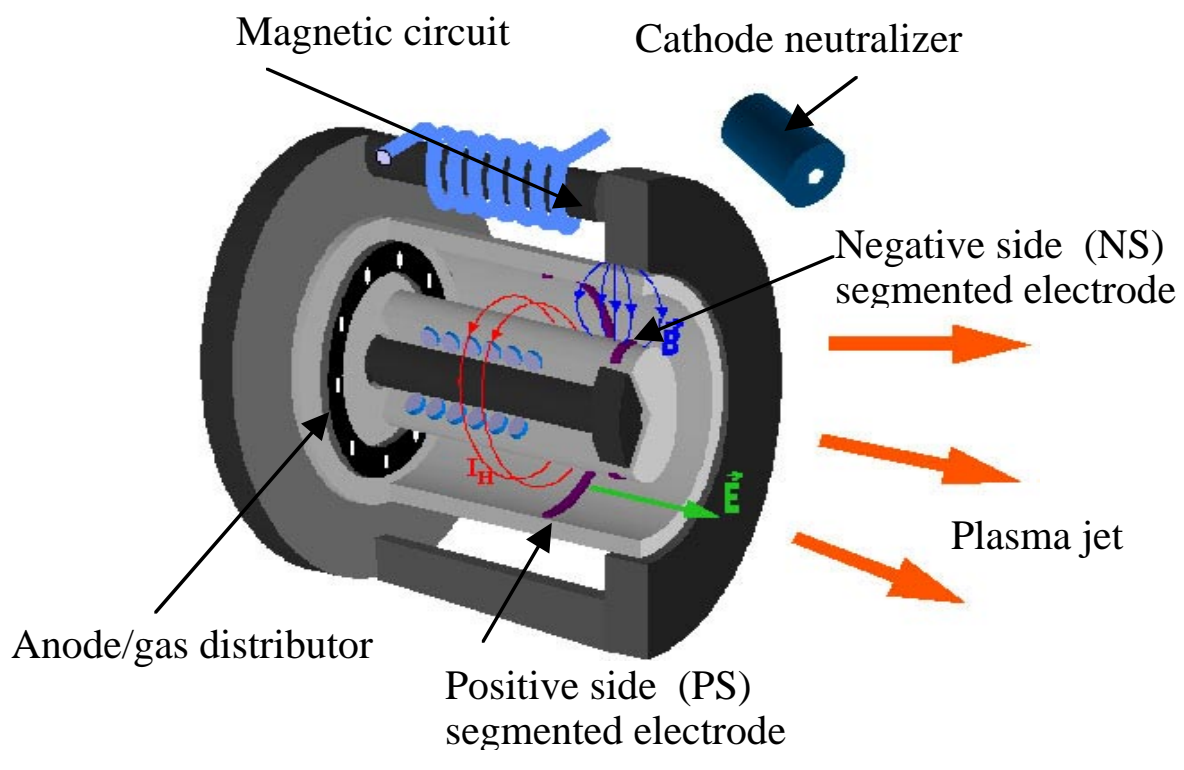

Fig. 1. A schematic drawing of Hall current accelerator with two segmented electrodes. E is the accelerating electric field, $\mathrm{B}$ is the applied magnetic field, $\mathrm{I}_{\mathrm{H}}$ is the electron Hall current.

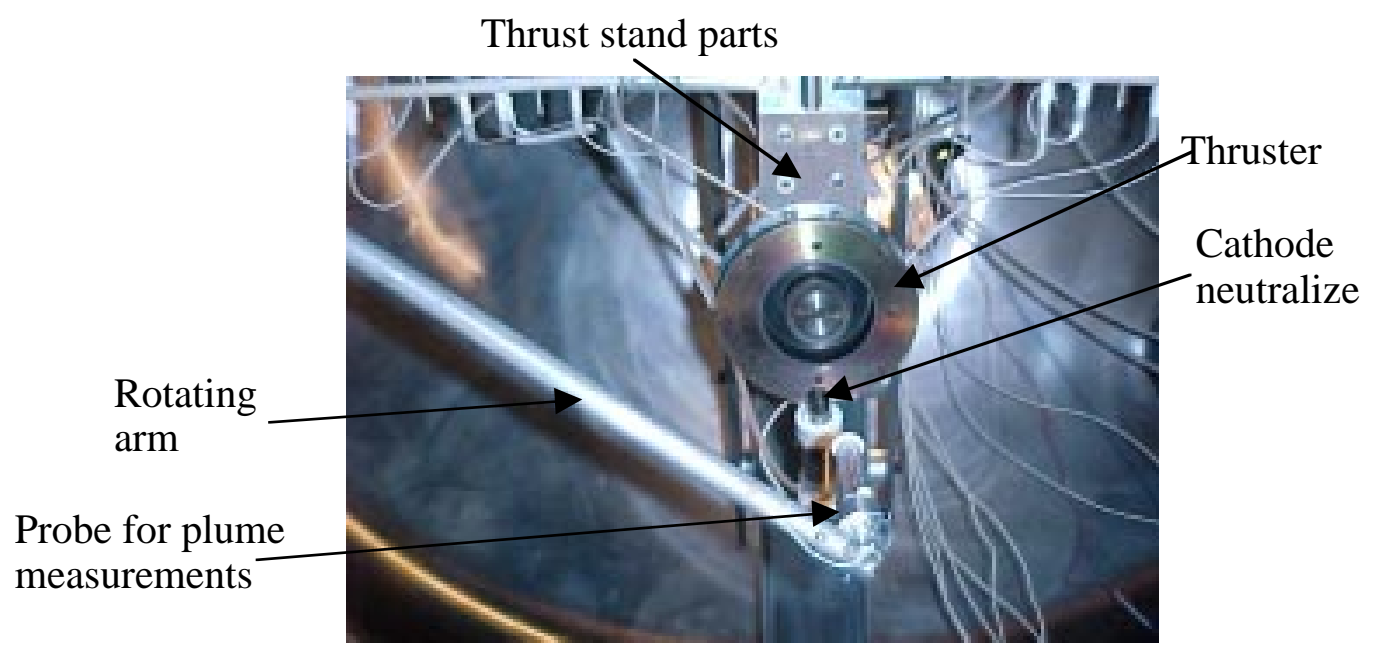

Fig. 2. PPPL segmented electrode Hall thruster. 


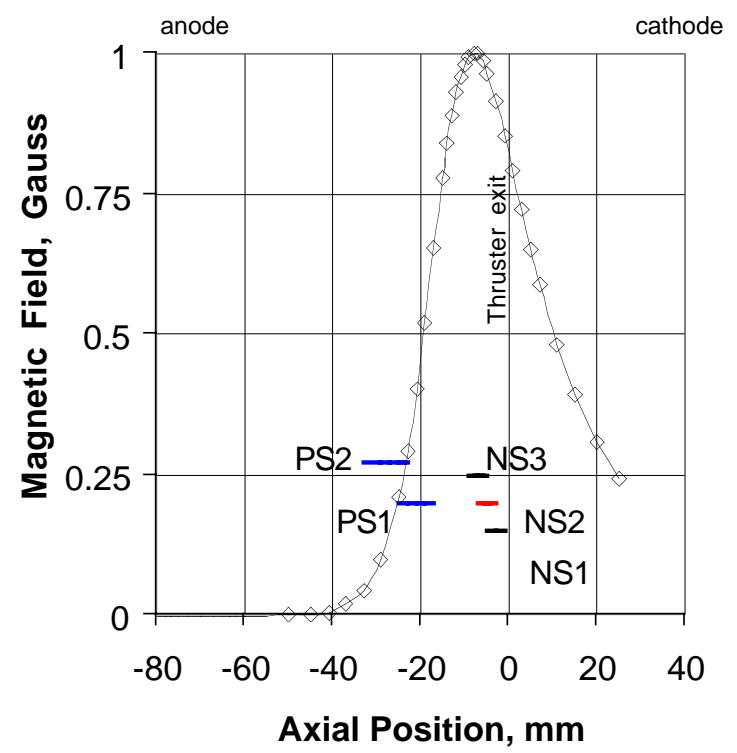

Fig. 3. Measured profile of the radial component of the magnetic field along the thruster axis near the channel median. Magnetic field is produced by a 2.1 A electromagnet current. NS1, NS2, NS3, PS1 and PS2 are locations of inner (negative side) and outer (positive side) segmented electrodes along thruster axis. 
(a)

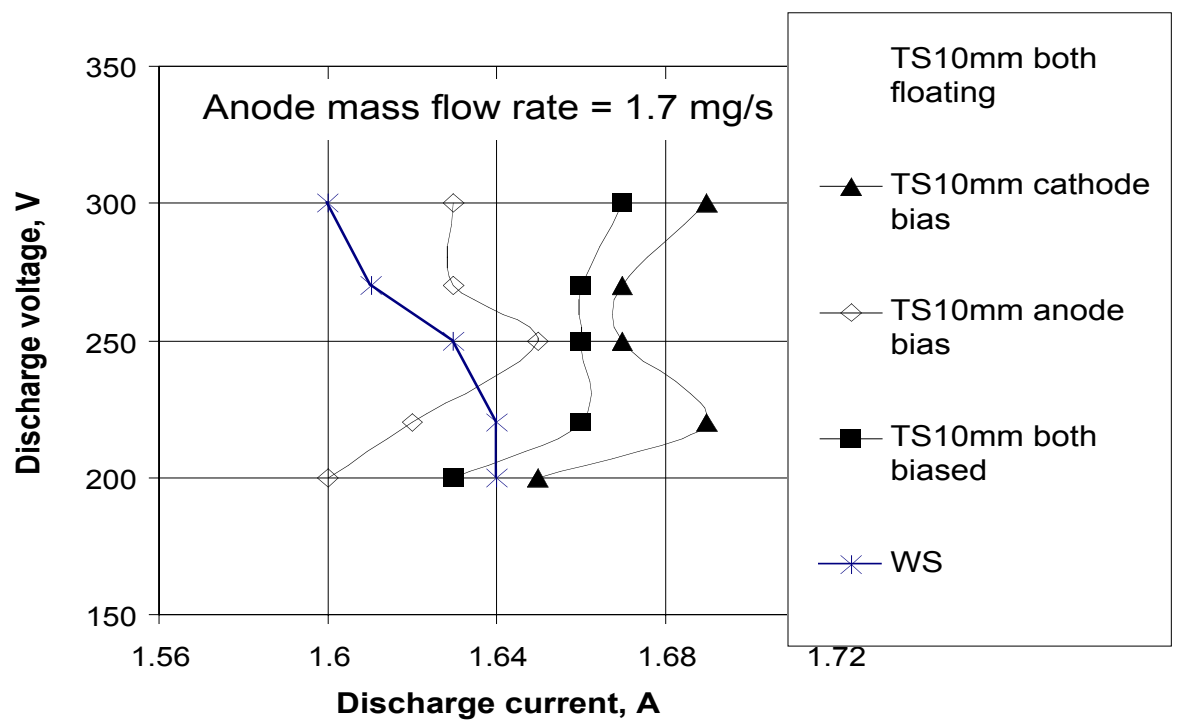

(b)

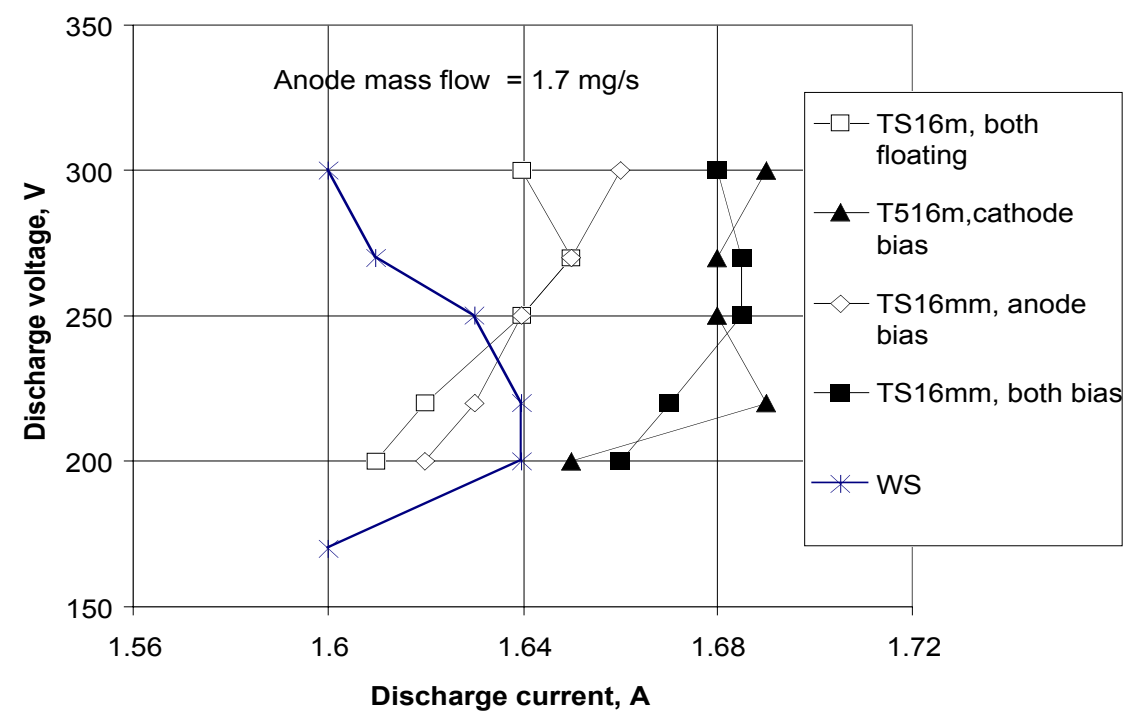

Fig. 4. Discharge voltage vs. discharge current at $1.7 \mathrm{mg} / \mathrm{s}$ for (a) $16 \mathrm{~mm}$ and (b) $10 \mathrm{~mm}$ spacing. TS is "two-segment" case, WS is "without segment" or conventional Hall thruster case. 
(a)

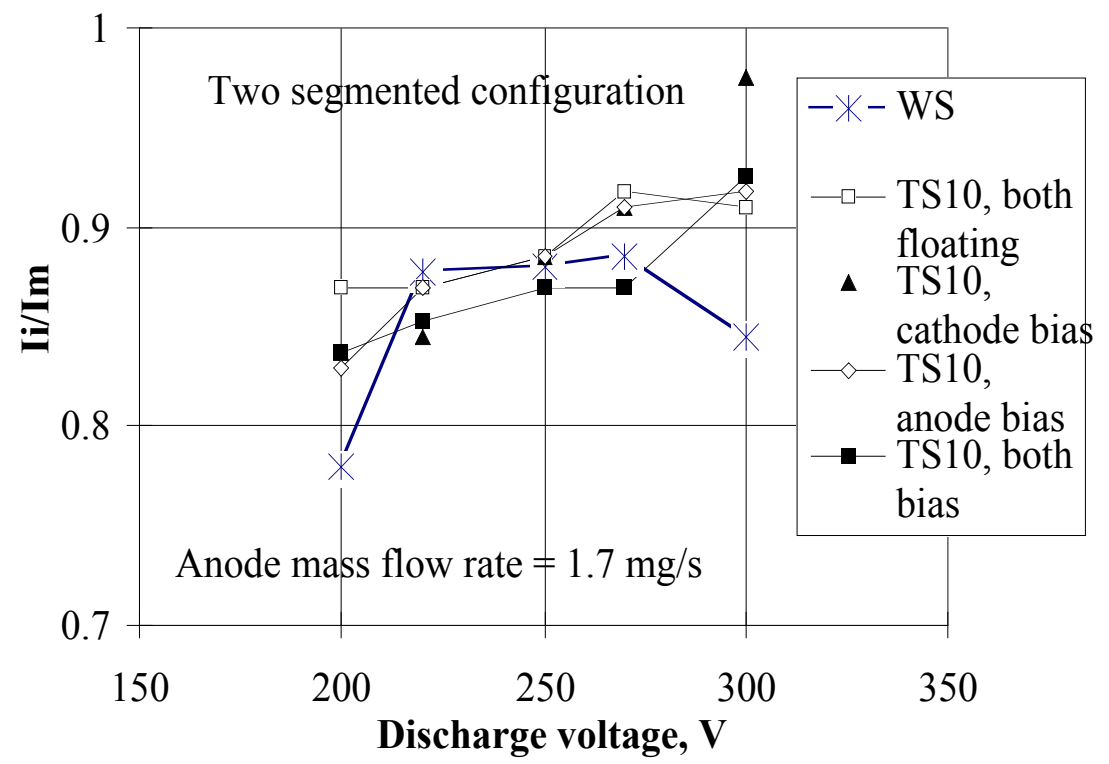

(b)

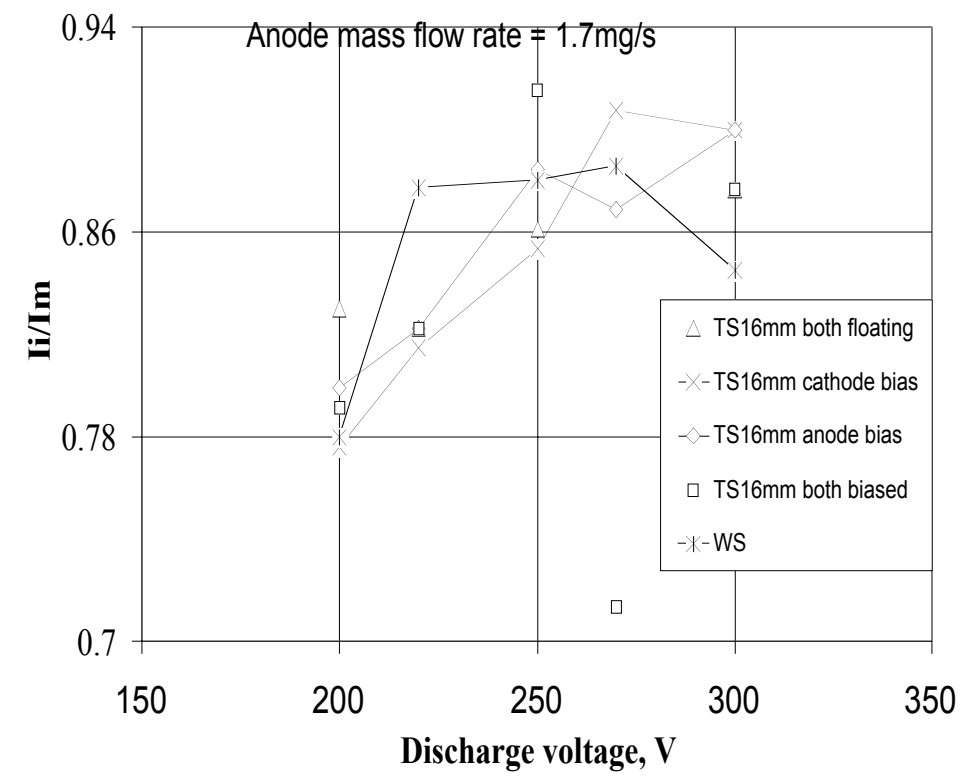

Fig. 5. Propellant utilization, $I_{i} / I_{m}$, where $I_{m}$ is equivalent flow rate in ampere units vs. discharge voltage (a) $16 \mathrm{~mm}$ spacing. (b) $10 \mathrm{~mm}$ spacing. 
(a)

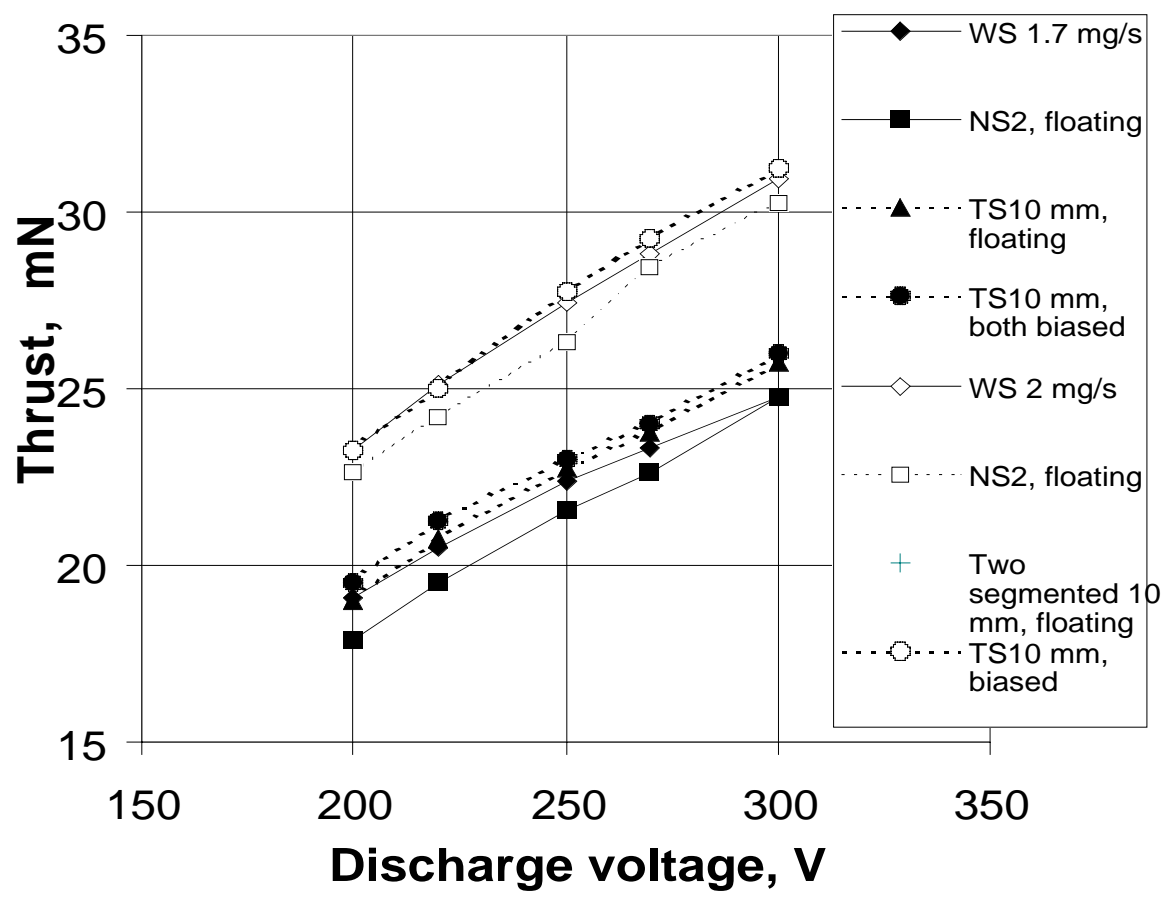

(b)

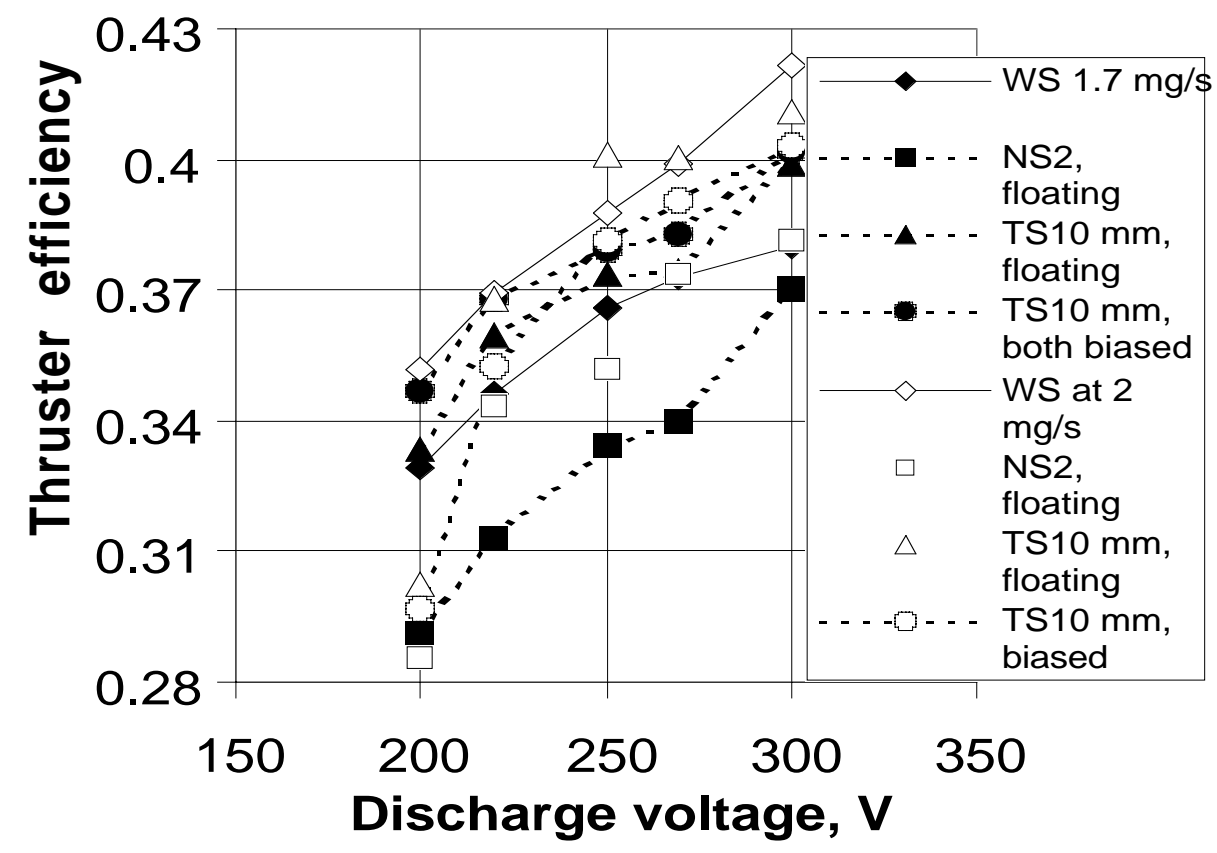

Fig. 6. Thrust (a) and the efficiency (b) versus discharge voltage at mass flow rate of 1.7 $\mathrm{mg} / \mathrm{s}$ measured for accelerator configurations with two segmented electrodes. 
(a)

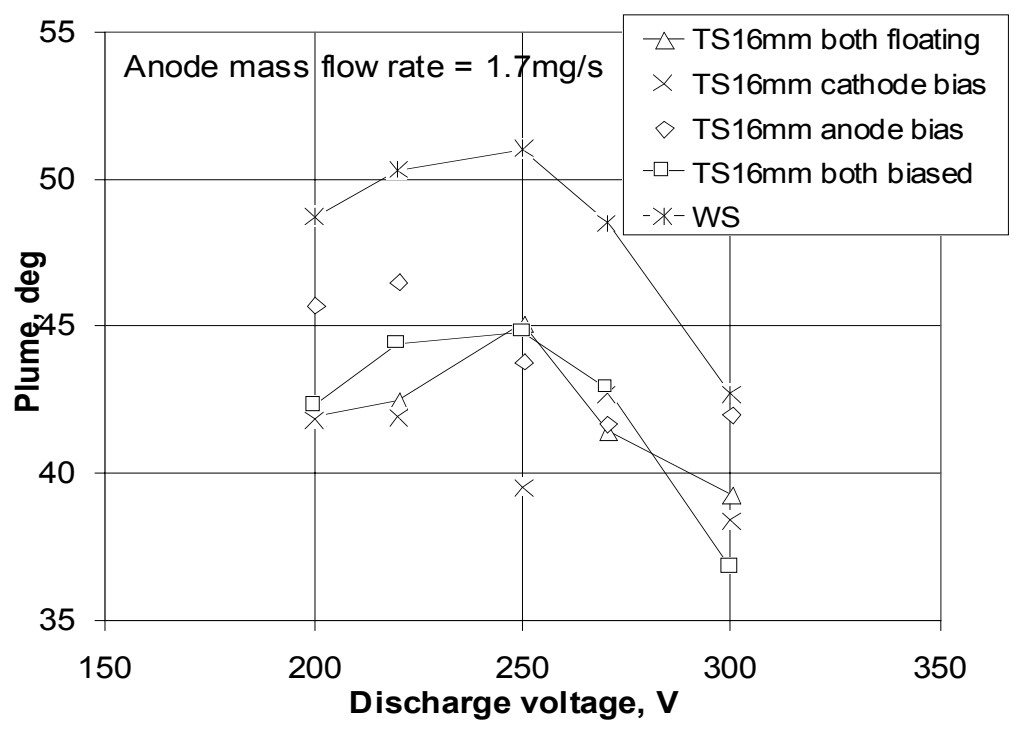

(b)

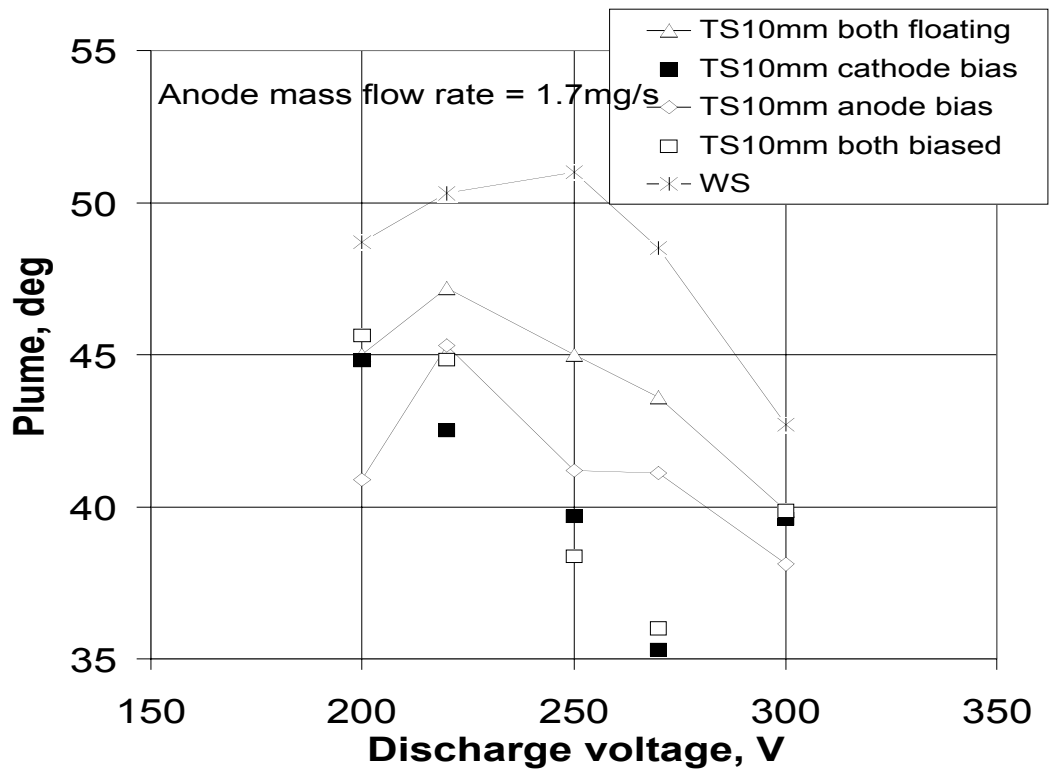

Fig. 7. Plume angle vs. discharge voltage at a mass flow rate of $1.7 \mathrm{mg} / \mathrm{s}$ :for two spacing between the electrodes (a) $10 \mathrm{~mm}$ (b) $16 \mathrm{~mm}$. 
(a)

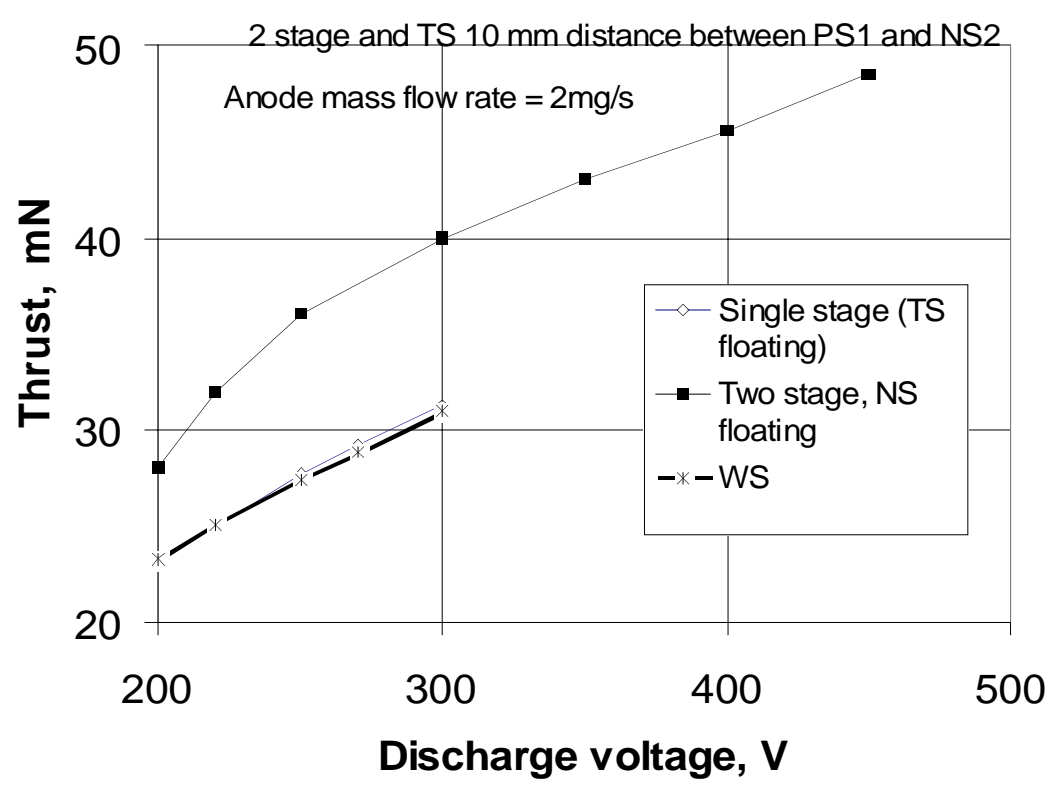

(b)

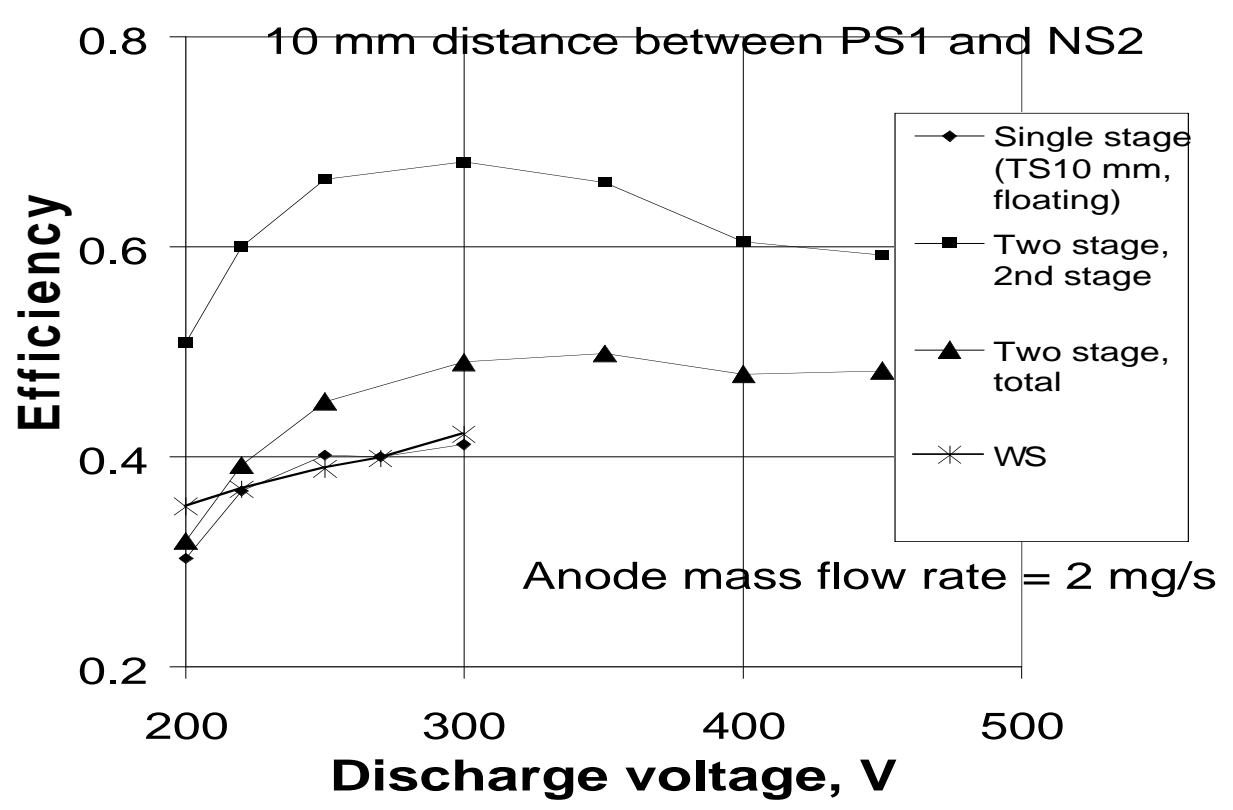

Fig. 8. Thrust (a) and the efficiency (b) versus discharge voltage measured for a twostage accelerator configuration at a mass flow rate of $2 \mathrm{mg} / \mathrm{s}$. 